\title{
What is the Evidence for a Link Between Digit Ratio (2D:4D) and Direct Measures of Prenatal Sex Hormones?
}

\author{
Gareth Richards, Ph.D. ${ }^{1}$ \\ ${ }^{1}$ Autism Research Centre, Department of Psychiatry, University of Cambridge, UK \\ * Corresponding author address: Autism Research Centre, University of Cambridge, Douglas House, 18b \\ Trumpington Road, Cambridge, CB2 8AH; email: gvr22@medschl.cam.ac.uk
}

\section{This is a pre-publication version of the following article:}

Richards, G. (2017) What is the evidence for a link between digit ratio (2D:4D) and direct measures of prenatal sex hormones? Early Human Development, 113, 71-72. https://doi.org/10.1016/j.earlhumdev.2017.08.003

Since Manning et al.'s [1] seminal paper was published in 1998, the ratio of length between the second (index) and fourth (ring) fingers (digit ratio, or 2D:4D) has been commonly employed by researchers as an indicator of prenatal sex hormone exposure. However, although an extensive literature now exists on how 2D:4D relates to a range of phenotypic outcomes, to the best of my knowledge there are currently only six papers that examine 2D:4D in relation to direct measures of prenatal sex hormones. Furthermore, the hormones reported on in four of these papers were measured from umbilical cord blood sampled shortly after birth. Although the blood might relate to foetal circulation in late gestation, the hormone levels measured technically relate to the perinatal rather than prenatal period (for a comparison of methods for measuring foetal sex hormones, see van de Beek et al. [2]).

Lutchmaya et al. [3] analysed foetal testosterone (fT) and oestradiol (fO) sampled from amniocentesis during the second trimester. Although left hand digit ratios (L2D:4D) in twoyear-olds were not associated with hormone levels, those for the right hand (R2D:4D) correlated negatively with the ratio of testosterone to oestradiol $(\beta=-0.007, p=0.004)$. However, the sample was small $(n=29)$, analyses for males and females were not conducted separately, and no other significant correlations were observed. It should however be noted that, although neither fT nor fO was a statistically significant predictor of digit ratio on its own, 
there was a trend level association between $\mathrm{fT}$ and $\mathrm{R} 2 \mathrm{D}: 4 \mathrm{D}(\beta=-3.74, p=0.09)$. If a onetailed test had been used, this effect would have been considered significant. This could arguably have been justified considering that a negative association between digit ratio and fT had already been hypothesised at this point [1]. A study of a separate cohort [4] reported that fT measured through amniocentesis correlated negatively with L2D:4D ( $r=-0.3, p=0.03$ ) and R2D:4D $(r=-0.24, p=0.09)$ in females $(n=51)$, although the latter effect was only marginally statistically significant, and no significant correlations were observed in males $(n=$ 49). As fO levels were not recorded in this study, no attempt could be made to replicate Lutchmaya et al.'s earlier finding.

Three papers have reported findings from subsamples of the Western Australian Pregnancy Cohort (Raine) Study, for which arterial and venous umbilical cord blood was sampled at birth. The first [5] found that foetal androgens and oestrogens (individually, or the ratio between them) did not predict digit ratio in adolescent females $(n=82)$. The second [6] reported similar non-significant findings for young adult males $(n=182)$ and females $(n=159)$. However, the third [7] found that bioavailable testosterone in females $(n=76)$ correlated negatively with L2D:4D $(r=-0.29, p<0.05)$, though no significant effect was observed for R2D:4D, and neither correlation was significant in males $(n=83)$. A possible reason for the discrepant findings here is that digit ratio was measured at different ages in each report (i.e. 14-16 years in [5], 19-22 years in [6], 21-24 years in [7]). In addition to the Raine cohort, a recent paper reported no significant associations between testosterone or oestrogen measured from umbilical cord blood, and digit ratio (for either hand) in a sample of 100 (50 male, 50 female) newborn children from Turkey [8].

Most reported correlations between 2D:4D and foetal sex hormones have not been statistically significant, although the literature broadly points towards a negative association with fT. However, it is interesting to note that no statistically significant effects have yet been reported in male-only samples. A potential explanation for this is that sex differences in hormonal activity are known to occur during normal foetal development. For instance, testosterone levels tend to peak between 14 and 18 weeks of gestation, with the largest sex differences being detected between 14 and 16 weeks (see [9,10]). Furthermore, testosterone is produced primarily by the testes in males and ovaries in females, though small amounts are also produced by the adrenal grands in both sexes. Of further interest is that both studies using amniotic fluid observed a significant effect, whereas only one significant effect was reported between the four 
studies of umbilical cord blood. A possible reason is that testosterone sampled from amniotic fluid and umbilical cord blood relates to the second and third trimesters, respectively, whereas development of the 2D:4D ratio may begin earlier, in the first trimester [11], with hormone concentrations in the later stages of gestation (i.e. the third trimester) having little or no influence on this trait.

Although there is experimental evidence for 2D:4D being influenced by prenatal sex hormones in animal studies (e.g. Zheng \& Cohn [12]), support for this hypothesised link in humans remains tenuous. For this reason, it is suggested that findings from studies involving 2D:4D as a proxy for foetal hormone exposure must be interpreted with caution. It should also be noted that studies of human amniotic fluid are scarce, and, due to various methodological advances, may not be replicated in the future. It would therefore be of considerable merit if existing study cohorts were followed up to better determine whether foetal sex hormone levels are predictive of adult digit ratios. This could help validate (or invalidate) digit ratio as a tool for examining the effects of the foetal environment on adult phenotype.

\section{References}

1. J.T. Manning, D. Scutt, J. Wilson, D.I. Lewis-Jones, The ratio of 2nd to 4th digit length: A predictor of sperm numbers and levels of testosterone, luteinizing hormone and oestrogen, Hum. Reprod. 13 (1998) 3000-3004. https://doi.org/10.1093/humrep/13.11.3000

2. C. van de Beek, J.H.H. Thijssen, P.T. Cohen-Kettenis, S.H.M. van Goozen, J.K. Buitelaar, Relationships between sex hormones assessed in amniotic fluid, and maternal and umbilical cord serum: What is the best source of information to investigate the effects of fetal hormonal exposure? Horm. Behav. 46 (2004) 663-669. http://doi.org/10.1016/j.yhbeh.2004.06.010

3. S. Lutchmaya, S. Baron-Cohen, P. Raggatt, R. Knickmeyer, J.T. Manning, 2nd to 4th digit ratios, fetal testosterone and estradiol, Early. Hum. Dev. 77 (2004) 23-28. https://doi.org/10.1016/j.earlhumdev.2003.12.002

4. T. Ventura, M.C. Gomes, A. Pita, M.T. Neto, A. Taylor, Digit ratio (2D:4D) in newborns: Influences of prenatal testosterone and maternal environment, Early. Hum. Dev. 89 (2013) 107-112. https://doi.org/10.1016/j.earlhumdev.2012.08.009

5. M. Hickey, D.A. Doherty, R. Hart, R.J. Norman, E. Mattes, H.C. Atkinson, D.M. Sloboda, Maternal and umbilical cord androgen concentrations do not predict digit ratio (2D:4D) in girls: A 
prospective cohort study, Psychoneuroendocrinology. $35 \quad$ (2010) 1235-1244. https://doi.org/10.1016/j.psyneuen. 2010.02.013

6. L.P. Hollier, J.A. Keelan, E.S.L Jamnadass, M.T. Maybery, M. Hickey, A.J.O Whitehouse, Adult digit ratio (2D:4D) is not related to umbilical cord androgen or estrogen concentrations, their ratios or net bioactivity, Early. Hum. Dev. 91 (2015), 111-117. https://doi.org/10. 1016/j.earlhumdev.2014.12.011.

7. A.J.O. Whitehouse, S.Z. Gilani, F. Shafait, A. Mian, D.W. Tan, M.T. Maybery, J.A. Keelan, R. Hart, D.J. Handelsman, M. Goonawardene, P. Eastwood, Prenatal testosterone exposure is related to sexually dimorphic facial morphology in adulthood, Proc. R. Soc. B Biol. Sci. 282 (2015), 20151351. https://doi.org/10.1098/rspb.2015.1351

8. R. Çetin, M. Can, E. Özcan, The relationship between testosterone and oestrogen level of the cord blood and length of fingers of newborns 2d: 4d. Balikesir Health Sci. J. 5 (2016) 75-82. https://doi.org/10.5505/bsbd.2016.49469

9. S. Baron-Cohen, S. Lutchmaya, R. Knickmeyer, Prenatal Testosterone in Mind, MIT Press, Cambridge, MA, 2004.

10. F.P. Prince, The triphasic nature of Leydig cell development in humans, and comments on nomenclature, J. Endocrinol. 168 (2001) 213-216. https://doi.org/10.1677/joe.0.1680213

11. M.A. Malas, S. Dogan, E.H. Evcil, K. Desdicioglu, Fetal development of the hands, digits and digit ratio (2D:4D), Early. Hum. Dev. 82 (2006) 469-475. https://doi.org/10.1016/j.earlhumdev.2005.12.002

12. Z. Zheng, M.J. Cohn, Developmental basis of sexually dimorphic digit ratios, Proc. Natl. Acad. Sci. 108 (2011) 16289-16294. https://doi.org/10.1073/pnas.1108312108 